\title{
Noise and Electrical Oscillations Generation during the Investigation of the Resistive Switching in the Yttria Stabilized Zirconia Films by Conductive Atomic Force Microscopy
}

\author{
Oleg Gorshkov, Dmitry Filatov, Dmitry Antonov, and Ivan Antonov \\ N. I. Lobachevsky State University of Nizhny Novgorod, 23 Gagarin Avenue, Building 3, Nizhny Novgorod 603950, Russia \\ Correspondence should be addressed to Dmitry Filatov; dmitry_filatov@inbox.ru
}

Received 11 September 2015; Accepted 20 October 2015

Academic Editor: Victor V. Moshchalkov

Copyright (C) 2015 Oleg Gorshkov et al. This is an open access article distributed under the Creative Commons Attribution License, which permits unrestricted use, distribution, and reproduction in any medium, provided the original work is properly cited.

The effect of resistive switching in the yttria stabilized zirconia (YSZ) thin films on Si substrates has been studied by Conductive Atomic Force Microscopy (CAFM). The resistive switching of the YSZ films from the low conductive state to the highly conductive one has been found to be associated with the increasing of the noise with broad frequency spectrum related to the redistribution of the oxygen vacancies in YSZ. The electrical oscillations in oscillation loop connected in series to the CAFM probe, the sample, and the bias source related to the excitation of the oscillation loop by the noise in the probe-to-sample contact film have been observed. The effect discovered is promising for application in the memristor devices of new generation.

\section{Introduction}

At present, the effect of resistive switching in the thin dielectric films has attracted increased interest of the researchers. The effect consists in reversible bistable change of the electrical resistance of thin dielectric films sandwiched between two conductive electrodes under the electric field induced by the bias voltage applied between the electrodes $[1,2]$. The resistive switching effect is expected to find wide field of application, particularly, in a new class of electronic devices called memristors [3], including the nonvolatile memory devices of the next generation [4].

Resistive switching effect has been observed in various materials like the transition metal oxides [5], chalcogenides [6], perovskites [7], and $\mathrm{SiO}_{x}$ [8] as well as in some polymers [9], and so forth. Yttria stabilized zirconia (YSZ) $\mathrm{ZrO}_{2}(\mathrm{Y}$ ) is considered to be promising material for the memristors due to high anion conductivity [10]. At present, the resistive switching effect on the metal oxides is considered to originate from the drift of the oxygen vacancies in the electric field induced between the conductive electrodes (which could be described also in terms of the drift of the oxygen ions via the vacancies) [11].

In the present study, the resistive switching effects in the thin $\left(\sim 5 \mathrm{~nm}\right.$ thick) YSZ films on the conductive $n^{+}-\mathrm{Si}$ substrates have been studied by Conductive Atomic Force Microscopy (CAFM) [12]. In this method, the surface of dielectric film on conductive substrate is scanned across by AFM probe with conductive coating. A bias voltage $V_{g}$ is applied between the AFM probe and the substrate, and the electric current via the probe-to-sample contact $I_{t}$ is measured as function of the probe coordinate on the sample surfaces $x$ and $y$ at $V_{g}=$ const. Otherwise, the currentvoltage $(I-V)$ curves of the probe-to-sample contact $I_{t}\left(V_{g}\right)$ are measured either at $x$ and $y=$ const or in every point of the AFM scan. Typical sizes of the contact area between the AFM probe tip and the sample surface in the contact AFM mode are 3 to $10 \mathrm{~nm}$ for typical loading forces $F_{n}=1$ to $2 \mathrm{nN}$ [13], which matches the expected sizes of the memory cells in future nonvolatile resistive memory (RRAM) devices $(\sim 10 \mathrm{~nm})$ [14]. So far, CAFM appears to be a powerful tool to study the resistive switching phenomena at the nanometer 


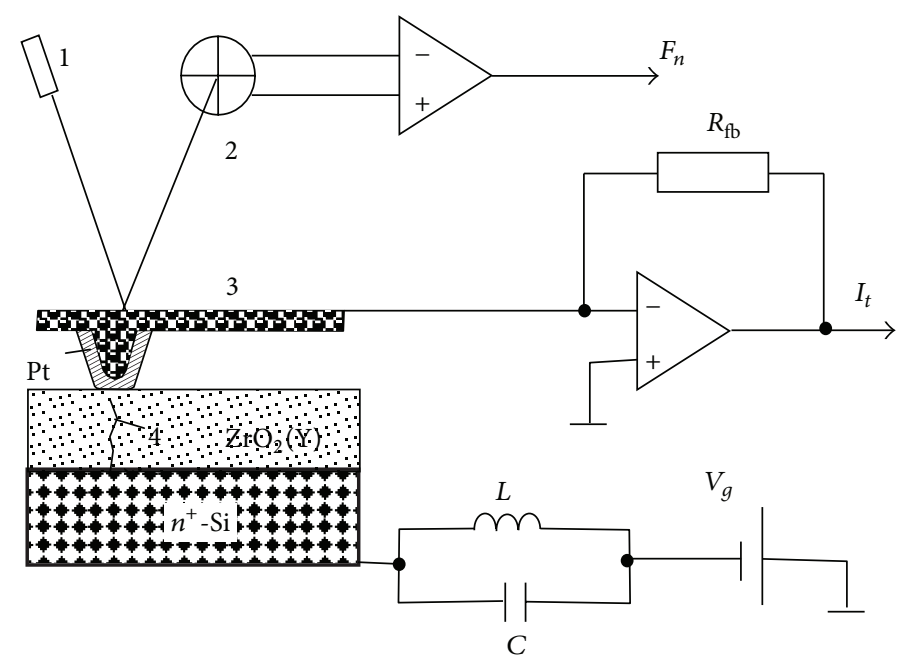

FIGURE 1: The schematic representation of the experiment on the investigation of the resistive switching effect in the YSZ/ $n^{+}-\mathrm{Si}$ films by Conductive Atomic Force Microscopy. 1-AFM laser; 2-quadrant photodetector; 3-Pt-coated cantilever; 4-filament.

scale. However, there were very few publications on studying the noise characteristics of the resistive switching phenomena using CAFM [15]. In these studies, the bandwidth of the analyzed noise was limited to $\sim 30 \mathrm{kHz}$. The goal of the present study was to investigate the noise characteristics of the resistive switching process in the thin $(\sim 5 \mathrm{~nm}$ thick) YSZ films on $\mathrm{Si}$ substrates in a broader frequency band ( 0 to $2 \mathrm{MHz}$ ) using CAFM.

\section{Materials and Methods}

The YSZ films of 3 to $5 \mathrm{~nm}$ in thickness were deposited onto the $n^{+}-\mathrm{Si}(001)$ substrates doped by arsenic with the specific resistivity $0.005 \mathrm{Ohm} \cdot \mathrm{cm}$ by Radio Frequency (RF) magnetron sputtering from composite $\mathrm{ZrO}_{2}-\mathrm{Y}_{2} \mathrm{O}_{3}$ compressed powder target in the $\mathrm{Ar}-\mathrm{O}_{2}$ gas mixture $(1: 1)$ using Torr International 2g1-1g2-eb4-th1 vacuum system for thin film deposition. The gas pressure in the deposition chamber was $\sim 10^{-2}$ Torr; the substrate temperature was $\approx 300^{\circ} \mathrm{C}$. The $\mathrm{Y}_{2} \mathrm{O}_{3}$ molar fraction in the target material was $\approx 0.12$.

The CAFM investigations were carried out in ultrahigh vacuum (UHV) environment at $300 \mathrm{~K}$ using UHV scanning tunneling microscope/atomic force microscope (STM/AFM) Omicron UHV STM/AFM LF1 installed into Omicron MultiProbe RM UHV system. The residual gas pressure in the STM/AFM chamber was $\sim 10^{-10}$ Torr. The schematic representation of the experiment is shown in Figure 1. The local electrical conductivity of the YSZ films was studied by measuring the electric current between the AFM probe and the Si substrate $I_{t}$ while scanning the sample surface in contact mode while applying the bias voltage $V_{g}$ between the AFM probe and the substrate. NT MDT NSG-11 $p^{+}-$Si AFM probes coated by Pt were used.

The investigation of the resistive switching in the YSZ/Si films was performed by recording the cyclic $I-V$ curves of the contact of the AFM probe to the YSZ film surface with the bias voltage sweep amplitude $V_{m}>V_{\text {th }}$, where $V_{\text {th }} \approx 4$ to $5 \mathrm{~V}$ was

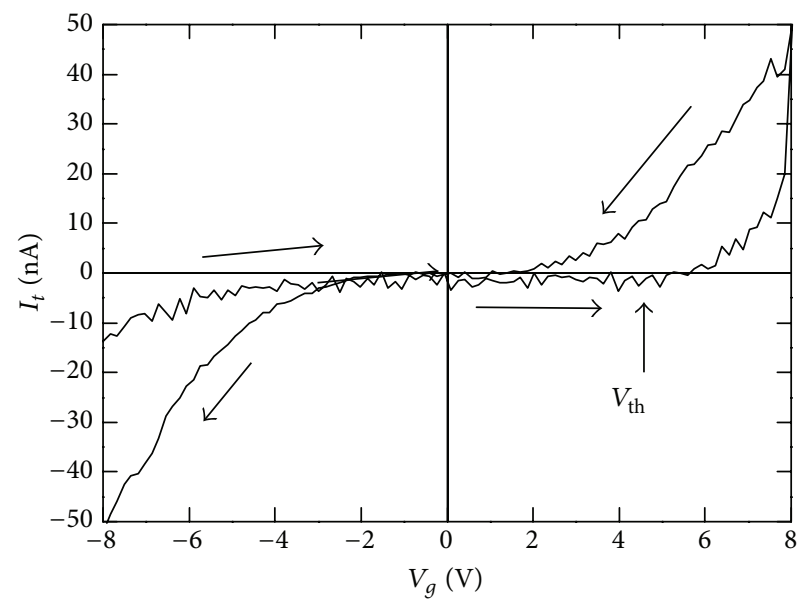

FIgUre 2: A typical cyclic $I-V$ curve of AFM probe contact to YSZ/Si film measured at $V_{m}=8 \mathrm{~V}$. The arrows denote the direction of $V_{g}$ sweep.

the threshold voltage for the resistive switching in the YSZ/Si films under study. The bias voltage between the probe and the substrate $V_{g}$ was increased linearly with increasing time $t$ from $-V_{m}$ up to $+V_{m}$ and then decreased back from $+V_{m}$ down to $-V_{m}$, and so on. The waveforms $I_{t}(t)$ were recorded using Agilent 3000 A oscilloscope connected to " $I_{t}$ " output of Omicron SCALA STM/AFM control electronics (Figure 1). More details on the sample preparation procedures as well as on the CAFM measurements ones can be found in [13].

\section{Results and Discussion}

The cyclic $I-V$ curves of the probe-to-sample contact measured on the YSZ/Si films have demonstrated well-expressed hysteresis of the bipolar type (Figure 2) due to the resistive switching in YSZ. The resistive switching behavior of the AFM tip contact to the YSZ/Si film was well reproducible. 


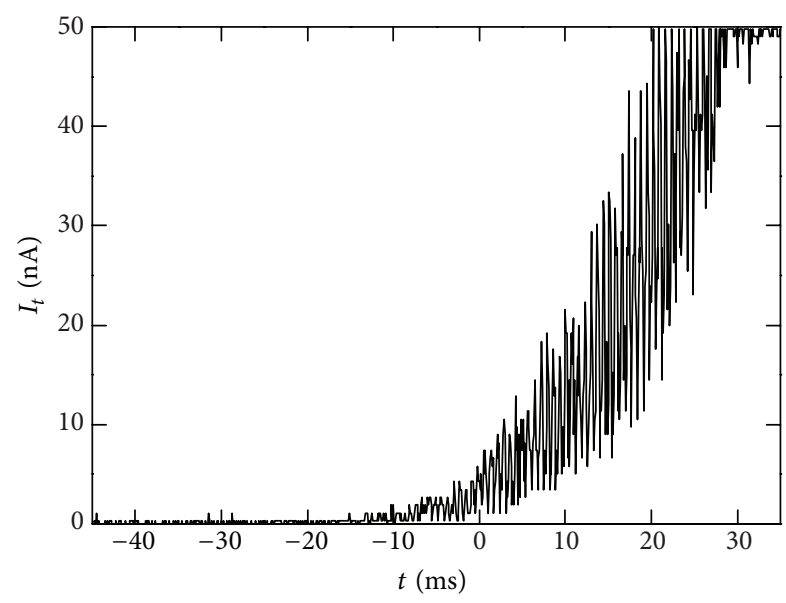

FIgURE 3: Typical probe current waveform $I_{t}(t)$ measured during the recording of $I-V$ curve of AFM probe contact to the YSZ/Si film surface at the linear sweep of $V_{g}$ in time from $0 \mathrm{~V}$ to $+8 \mathrm{~V}$.

Earlier, in a dedicated study of the resistive switching in the YSZ/Si films by CAFM [13] we have observed the reproducible cyclic $I-V$ curves with hysteresis like the one presented in Figure 2 when up to several hundred cyclic $I$ $V$ curves were measured in one point of the sample surface.

A typical probe current waveform $I_{t}(t)$ recorded during the measurement of $I-V$ curve when sweeping $V_{g}$ from $0 \mathrm{~V}$ up to $+8 \mathrm{~V}$ is presented in Figure 3. The increase of $I_{t}$ at $V_{g} \approx V_{\text {th }} \approx 4 \mathrm{~V}$ was related to the switching of the YSZ film from the initial low-conductive ("OFF") state into the highly conductive ("ON") one. One can see in Figure 3 that the increase of $I_{t}$ due to the resistive switching in the YSZ film is associated with the increased noise at $V_{g}>V_{\text {th }} \approx 4 \mathrm{~V}$. The noise generation has been observed in all points of the sample surface. Earlier, we have studied the electron tunneling via the tunnel transparent $\mathrm{SiO}_{2} / \mathrm{Si}$ films with the embedded $\mathrm{Au}$ nanoparticles [16, 17], via the $\mathrm{In}(\mathrm{Ga}) \mathrm{As} / \mathrm{GaAs}(001)$ quantum dots and rings $[18,19]$, and via the $\mathrm{GeSi} / \mathrm{Si}(001)$ self-assembled nanoislands $[19,20]$ by CAFM. We have not observed such noise generation in the objects listed above as in the YSZ/Si films. On the other hand, the $\mathrm{SiO}_{2}$ :nc-Au films have not exhibited the resistive switching (no hysteresis has been observed in the $I-V$ curves). Therefrom, we have concluded the noise generation in the YSZ/Si films to be related directly to the resistive switching property of these ones and suggested both phenomena to have the same physical origin.

As it was already mentioned above, today's understanding of the resistive switching mechanism in the transition metal oxides with high anion mobility is based on the concept of the oxygen ion drift via the oxygen vacancies in the external electric field (which could be also treated as the drift of the vacancies in the opposite direction) [11]. During the forming process, the oxygen vacancies arrange in the chains (so-called filaments) growing through almost whole dielectric film under the external electric field [21]. The oxygen vacancies in YSZ generate the deep donor energy levels with the excitation energy $\approx 0.3 \mathrm{eV}[10]$. The electron conductivity via the filaments originates from the hopping of electrons between the adjacent vacancy deep levels; in the ultimate limit, the overlap of the envelope wavefunctions of the deep level states related to the oxygen vacancies in the nearest neighbor sites arranged in a chain generates a one-dimensional electron miniband [22]. Within the framework of this concept, the resistive switching in the metal-dielectric-metal (MDM) stacks as well as in the metal-oxide-semiconductor (MOS) ones from the "ON" state to the "OFF" one is related to the destruction of the filament near one of the metal/dielectric interfaces with formation of a vacancy-free blocking dielectric layer between the filament tip and the metal-dielectric interface because of the drift of the oxygen ions towards the interface. There, these ones fill the oxygen vacancies thus blocking the electron conductivity via the vacancyrelated deep levels [23]. The reverse switching is related to the destruction of the blocking layer because of attraction of the oxygen vacancies to the tip of the filament (or, in other words, because of the drift of the oxygen ions away from the blocking layer) that restores the filaments until the blocking layer becomes a tunnel transparent one [24].

As it has been already mentioned above, typical size of the contact area between the AFM probe tip and the sample surface is $<10 \mathrm{~nm}$ [13]. On the other hand, the highest surface density of the filaments in the MDM memory cells reported in the literature was $<10^{12} \mathrm{~cm}^{-2}$ [25]. So far, the maximum number of filaments to appear under the contact of the AFM probe tip to the sample surface makes 1 to 2 . At the same time, the thickness of the blocking layer is also rather small: in order to restore the low-conductive ("OFF") state, the blocking layer is enough to become a tunnel nontransparent one. When increasing the thickness of the blocking layer in $\approx 0.25 \mathrm{~nm}$ the tunnel current through this one decreases by order of magnitude [26]. So far, when the blocking layer thickness is $\sim 1 \mathrm{~nm}$, the conductivity of the filament is reduced by a factor of $\sim 10^{4}$ as compared to the case when the filament grows through the film completely.

Thus, within the framework of this concept, the electromigration processes of the oxygen ions responsible for the resistive switching in the contact of AFM probe to the YSZ film surface take place in a very small volume that could be estimated as $\sim 10 \times 10 \times 1 \mathrm{~nm}^{3}$. In this case, when the ion drift path becomes comparable to the YSZ lattice constant, a discrete character of the oxygen ion electromigration becomes an essential one. Namely, a single jump of an oxygen ion to the neighbor vacancy located near the tip of a filament can affect the probe current $I_{t}$ considerably. Thus, as it has been shown in [22], the filling of a single vacancy in a one-dimensional chain by an oxygen ion results in the decrease of the filament conductivity by more than an order of magnitude. So far, the above speculations allow relating the increased noise in the resistive switching of the contact of AFM probe to the YSZ/Si film surface to a discrete essentially, on one hand, and, on the other hand, a stochastic nature of the oxygen ion electromigration via the vacancies under the AFM tip contact to the YSZ film surface. In other words, the nature of the observed noise is similar to the one of the shot noise.

In Figure 4, a typical waveform of the noise signal recorded in the process of the resistive switching with 


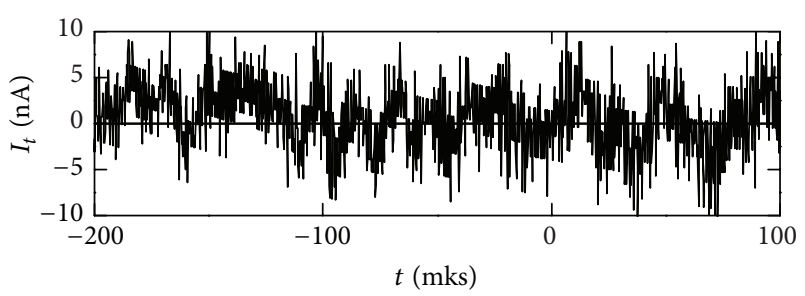

(a)

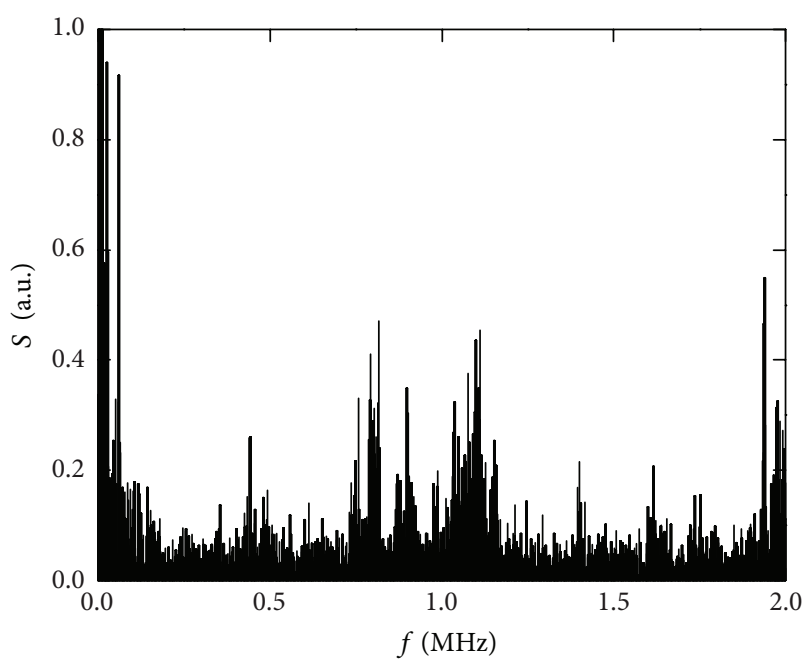

(b)

Figure 4: A waveform (a) and the power spectrum (b) of noise signal generated during the resistive switching in AFM probe contact to the YSZ/Si film.

increased time resolution (the sampling time was set to $250 \mathrm{~ns}$ ) after the trend subtraction (a) as well as its normalized power spectrum calculated by fast Fourier transform (b) is presented. The flicker noise band with the spectral power density $S(f)$ decreasing rapidly with increasing frequency $f$ has been observed in the lower frequency part of the spectrum. Earlier, $1 / f$ noise in the MDM stacks based on the bilayer $\mathrm{ZrO}_{x} / \mathrm{HfO}_{x}$ films has been reported [27]. The authors of [27] attributed this $1 / f$ noise to the trap-assisted tunneling of the electrons in the electron transport via the filaments.

Besides the flicker noise band, one can see a nearly uniform band typical for the shot noise in the higher frequency part of the spectrum. In addition, one can see several peaks in the noise spectrum near $f \approx 1$ and $2 \mathrm{MHz}$, which can be related to some characteristic lifetime of oxygen ion with respect to jump onto adjacent vacancy $\tau$. Assuming the temperature dependence of $\tau$ to obey Arrhenius law

$$
\tau=\tau_{0} \exp \left(-\frac{E_{a}}{k T}\right)
$$

where $\tau_{0} \sim 1 / \nu_{0}, v_{0} \sim 10^{13} \mathrm{~Hz}$, is the phonon frequency, $k$ is Boltzmann constant, $T$ is the temperature, and $E_{a}$ is the activation energy for the oxygen ion jump, for $f=1 \mathrm{MHz}$ one gets $\tau \sim 1 / f \sim 10^{-6} \mathrm{~s}$, and for $T=300 \mathrm{~K}$ one gets $E_{a} \approx 0.4 \mathrm{eV}$. This estimate is more than 2 times lower than the typical values of the activation energy for the ion conductivity in the YSZ thin films at low temperatures $\left(T<900^{\circ} \mathrm{C}\right)$ reported in the literature ( 0.8 to $1.1 \mathrm{eV}$, subject to $\mathrm{Y}$ fraction and to the thin film preparation method $[28,29])$.

This discrepancy can be attributed to the following two factors. The first one is the thermal heating of the filament [23]. Indeed, according to (1), the value of $E_{a}=0.8 \mathrm{eV}$ for $\tau=10^{-6} \mathrm{~s}$ and $\tau_{0}=10^{-13} \mathrm{~s}$ corresponds to $T \approx 600 \mathrm{~K}$ which seems reasonable. Another factor is the decrease of the activation energy in the external electric field. According to Poole-Frenkel formula [30], the decrease of $E_{a}$ in the electric field of the strength $F$ can be estimated as

$$
\Delta E \sim e \sqrt{\frac{e F}{4 \pi \varepsilon \varepsilon_{0}}},
$$

where $e$ is the elementary charge, $\varepsilon$ is the relative dielectric permittivity of YSZ, and $\varepsilon_{0}$ is the electric constant. According to (2), for $\Delta E=0.4 \mathrm{eV}$ and $\varepsilon=20$ for YSZ [10] one gets an estimate of the electric field strength in the blocking layer between the filament tip and the contact of the AFM tip coating to the YSZ film surface $F \approx 2 \times 10^{7} \mathrm{~V} / \mathrm{cm}$. Therefrom, assuming the electric potential of the filament core to be close to the one of the $n^{+}$-Si substrate along the whole filament length, one gets an estimate of the blocking layer thickness $d \sim V_{\mathrm{th}} / F \approx 2 \mathrm{~nm}$ for $V_{\mathrm{th}}=4 \mathrm{~V}$ that is of the same order of magnitude as the estimate of the blocking layer thickness given above.

The hypothesis on the origin of the noise generation during the resistive switching in the contact of the AFM probe to the YSZ film surface presented above allows also explaining why such noise has not been observed earlier in the MDM cells with the macroscopic electrode sizes. In such cells, the macroscopic electrodes cover a considerable number of filaments as a rule. For example, assuming the surface density of filaments $N_{s} \sim 10^{12} \mathrm{~cm}^{-2}$ [25] one has $\sim 10^{4}$ filaments under an electrode of $1 \times 1 \mu \mathrm{m}$ in size, so that the shot noise is eliminated because of averaging of the electric current flowing through different filaments. On the contrary, in the case of the nanometer size of the electrodes (which takes place in the CAFM experiments) when the AFM probe covers a few filaments, the discrete character of the oxygen ion electromigration becomes essential. Therefore, the noise generation during the resistive switching could affect the operation of the future memristor devices with the expected electrode dimensions of the order of $\sim 10 \mathrm{~nm}$ [14] essentially that should be taken into account in the design of these ones.

On the other hand, the discovered effect of the noise generation can be applied to various novel memristor devices. In Figure 5, a probe current waveform $I_{t}(t)$ recorded at $V_{g}=$ $6 \mathrm{~V}$, that is, at the maximum noise amplitude, is presented. At that, an oscillating loop consisting of a coil $L$ and a capacitor $C$ was connected to the bias source, the sample, the AFM probe, and the STM preamplifier (current-voltage converter) in series (Figure 1). The resonant frequency of the oscillating loop was selected to be $\approx 24 \mathrm{kHz}$. The observed sinusoidal oscillations were attributed to the excitation of the oscillating loop by the noise in the AFM probe-to-sample contact during the resistive switching of the YSZ film. The effect of the sinusoidal oscillations generation on the oscillating loop 


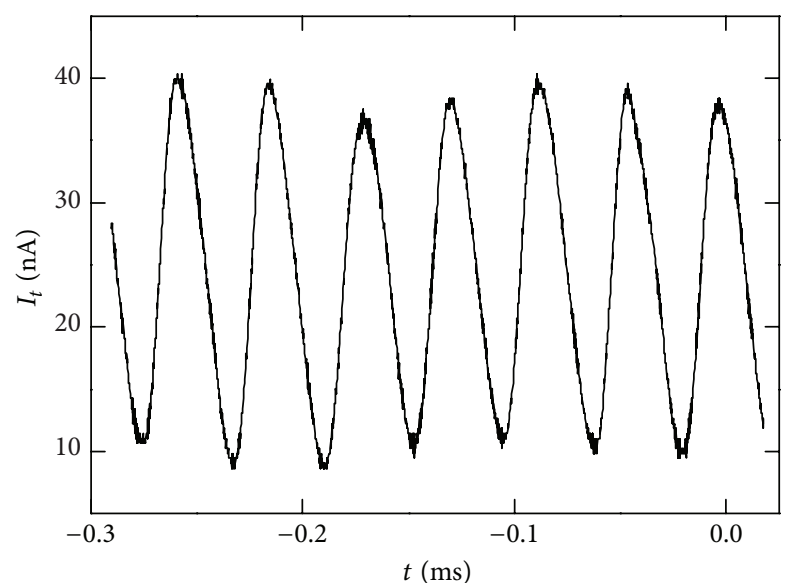

FIGURE 5: A waveform of the probe current oscillations measured when oscillating loop is connected into the AFM probe-YSZ/Si filmbias source circuit (Figure 1).

can find various applications in the memristor devices, for example, to detect the moment of the resistance switching in the MDM cells.

\section{Conclusion}

In the present study, the effect of the noise generation during the resistive switching on the contact of a conductive AFM probe to YSZ/Si films has been observed. The noise was attributed to the essentially discrete nature of the oxygen ion electromigration in the YSZ films under the external electric field between the AFM probe and the conductive Si substrate. The discovered effect can find various applications in the future memristor devices. Particularly, the generation of the sinusoidal electrical oscillations in the oscillating loop connected to the AFM probe and the YSZ/Si structure in series has been observed in the present study. The oscillations were attributed to the excitation of the oscillating loop by the noise in the probe-to-sample contact.

\section{Conflict of Interests}

The authors declare that there is no conflict of interests regarding the publication of this paper.

\section{Acknowledgments}

The present work has been supported by Ministry of Education and Science, Russian Federation (3.2441.2014/K). The CAFM experiments have been carried out using the shared research facilities of Research and Educational Center for Physics of Solid State Nanostructures, N. I. Lobachevsky State University of Nizhny Novgorod.

\section{References}

[1] R. Waser and M. Aono, "Nanoionics-based resistive switching memories," Nature Materials, vol. 6, no. 11, pp. 833-840, 2007.
[2] R. Waser, "Resistive non-volatile memory devices," Microelectronic Engineering, vol. 86, no. 7-9, pp. 1925-1928, 2009.

[3] D. B. Strukov, G. S. Snider, D. R. Stewart, and R. S. Williams, "The missing memristor found," Nature, vol. 453, pp. 80-83, 2008.

[4] D. S. Jeong, R. Thomas, R. S. Katiyar et al., "Emerging memories: resistive switching mechanisms and current status," Reports on Progress in Physics, vol. 75, no. 7, Article ID 076502, 2012.

[5] A. Sawa, "Resistive switching in transition metal oxides," Materials Today, vol. 11, no. 6, pp. 28-36, 2008.

[6] M. N. Kozicki, M. Yun, L. Hilt, and A. Singh, "Applications of programmable resistance changes in metal-doped chalcogenides," Journal of Electrochemical Society, vol. 146, no. 1, pp. 298-309, 1999.

[7] T. Baiatu, R. Waser, and K.-H. J. Haerdtl, "DC electrical degradation of perovskite-type titanates: III, a model of the mechanism," Journal of the American Ceramic Society, vol. 73, no. 6, pp. 1663-1673, 1990.

[8] T.-Y. Lin, L.-M. Chen, S.-C. Chang, and T.-S. Chin, "Electrical resistance switching in $\mathrm{Ti}$ added amorphous $\mathrm{SiO}_{\mathrm{x}}$ " Applied Physics Letters, vol. 95, no. 16, Article ID 162105, 2009.

[9] J. Y. Ouyang, C.-W. Chu, C. R. Szmanda, L. P. Ma, and Y. Yang, "Programmable polymer thin film and non-volatile memory device," Nature Materials, vol. 3, no. 12, pp. 918-922, 2004.

[10] H. A. Abbas, Stabilized Zirconia for Solid Oxide Fuel Cells or Oxygen Sensors: Characterization of Structural and Electrical Properties of Zirconia Doped with Some Oxides, LAP Lambert Academic, 2012.

[11] R. Waser, R. Dittmann, C. Staikov, and K. Szot, "Redoxbased resistive switching memories nanoionic mechanisms, prospects, and challenges," Advanced Materials, vol. 21, no. 2526, pp. 2632-2663, 2009.

[12] M. Lanza, "A review on resistive switching in high-k dielectrics: a nanoscale point of view using conductive atomic force microscope," Materials, vol. 7, no. 3, pp. 2155-2182, 2014.

[13] D. O. Filatov, D. A. Antonov, O. N. Gorshkov et al., "Investigation of resistive switching in the nanocomposite zirconia films by tunneling atomic force microscopy," in Atomic Force Microscopy (AFM): Principles, Modes of Operation and Limitations, pp. 335-355, Nova Science Publishers, New York, NY, USA, 2014.

[14] H.-S. P. Wong, H.-Y. Lee, S. Yu et al., "Metal-oxide RRAM," Proceedings of the IEEE, vol. 100, no. 6, pp. 1951-1970, 2012.

[15] J.-O. Krisponeit, C. Kalkert, B. Damaschke, V. Moshnyaga, and K. Samwer, "Time-resolved resistive switching on manganite surfaces: creep and $1 / f^{\alpha}$ noise signatures indicate pinning of nanoscale domains," Physical Review B, vol. 87, no. 12, Article ID 121103(R), 2013.

[16] D. O. Filatov, M. A. Lapshina, D. A. Antonov, O. N. Gorshkov, A. V. Zenkevich, and Y. Y. Lebedinskii, "Resonant tunnelling through individual $\mathrm{Au}$ nanoclusters embedded in ultrathin $\mathrm{SiO}_{2}$ films studied by tunnelling AFM," Journal of Physics: Conference Series, vol. 245, Article ID 012018, 2010.

[17] A. Zenkevich, Y. Lebedinskii, O. Gorshkov, D. Filatov, and D. Antonov, "Structural and electron transport properties of ultrathin $\mathrm{SiO}_{2}$ films with embedded metal nanoclusters grown on Si," in Advances in Diverse Industrial Applications of Nanocomposites, B. Reddy, Ed., chapter 14, pp. 317-340, InTech, Rijeka, Croatia, 2011.

[18] D. O. Filatov, M. A. Lapshina, M. A. Isakov, P. A. Borodin, and A. A. Bukharaev, "Tunnelling AFM study of the local density of 
states in the self assembled $\operatorname{In}(\mathrm{Ga}) \mathrm{As} / \mathrm{GaAs}(001)$ quantum dots and rings," Journal of Physics: Conference Series, vol. 244, no. 1, Article ID 012017, 2010.

[19] D. Filatov, V. Shengurov, N. Nurgazizov, P. Borodin, and A. Bukharaev, "Tunneling atomic force microscopy of selfassembled $\operatorname{In}(\mathrm{Ga}) \mathrm{As} / \mathrm{GaAs}$ quantum dots and rings and of $\mathrm{GeSi} / \mathrm{Si}(001)$ nanoislands," in in Fingerprints in the Optical and Transport Properties of Quantum Dots, A. Al-Ahmadi, Ed., chapter 12, pp. 273-298, InTech, Rijeka, Croatia, 2012.

[20] P. A. Borodin, A. A. Bukharaev, D. O. Filatov et al., "Investigation of the local density of states in self-assembled $\mathrm{GeSi} / \mathrm{Si}(001)$ nanoislands by combined scanning tunneling and atomic-force microscopy," Semiconductors, vol. 45, no. 3, pp. 403-407, 2011.

[21] K. M. Kim, D. S. Jeong, and C. S. Hwang, "Nanofilamentary resistive switching in binary oxide system; A review on the present status and outlook," Nanotechnology, vol. 22, no. 25, Article ID 254002, 2011.

[22] X. Lian, X. Cartoixà, E. Miranda et al., "Multi-scale quantum point contact model for filamentary conduction in resistive random access memories devices," Journal of Applied Physics, vol. 115, no. 24, Article ID 244507, 2014.

[23] Y. S. Lin, F. Zeng, S. G. Tang et al., "Resistive switching mechanisms relating to oxygen vacancies migration in both interfaces in Ti/ $\mathrm{HfO}_{x} / \mathrm{Pt}$ memory devices," Journal of Applied Physics, vol. 113, no. 6, Article ID 064510, 2013.

[24] J. J. Yang, D. B. Strukov, and D. R. Stewart, "Memristive devices for computing," Nature Nanotechnology, vol. 8, no. 1, pp. 13-24, 2013.

[25] M. Rogala, G. Bihlmayer, W. Speier, Z. Klusek, C. Rodenbücher, and K. Szot, "Resistive switching of a quasi-homogeneous distribution of filaments generated at heat-treated $\mathrm{TiO}_{2}$ (110)surfaces," Advanced Functional Materials, vol. 25, no. 40, pp. 6382-6389, 2015.

[26] S. Yu, X. Guan, and H.-S. P. Wong, "Conduction mechanism of TiN/ $/ \mathrm{HfO}_{\mathrm{x}} / \mathrm{Pt}$ resistive switching memory: a trap-assistedtunneling model," Applied Physics Letters, vol. 99, Article ID 063507, 2011.

[27] D. Lee, J. Lee, M. Jo, J. Park, M. Siddik, and H. Hwang, "Noiseanalysis-based model of filamentary switching ReRAM with $\mathrm{ZrO}_{x} / \mathrm{HfO}_{x}$ Stacks," IEEE Electron Device Letters, vol. 32, no. 7, pp. 964-966, 2011.

[28] I. Kosacki, V. Petrovsky, and H. U. Anderson, "Modeling and characterization of electrical transport in oxygen conducting solid electrolytes," Journal of Electroceramics, vol. 4, no. 1, pp. 243-249, 2000.

[29] C. Zhang, C.-J. Li, G. Zhang et al., "Ionic conductivity and its temperature dependence of atmospheric plasma-sprayed yttria stabilized zirconia electrolyte," Materials Science and Engineering B: Solid-State Materials for Advanced Technology, vol. 137, no. 1-3, pp. 24-30, 2007.

[30] J. Frenkel, "On pre-breakdown phenomena in insulators and electronic semi-conductors," Physical Review, vol. 54, no. 8, pp. 647-648, 1938 . 

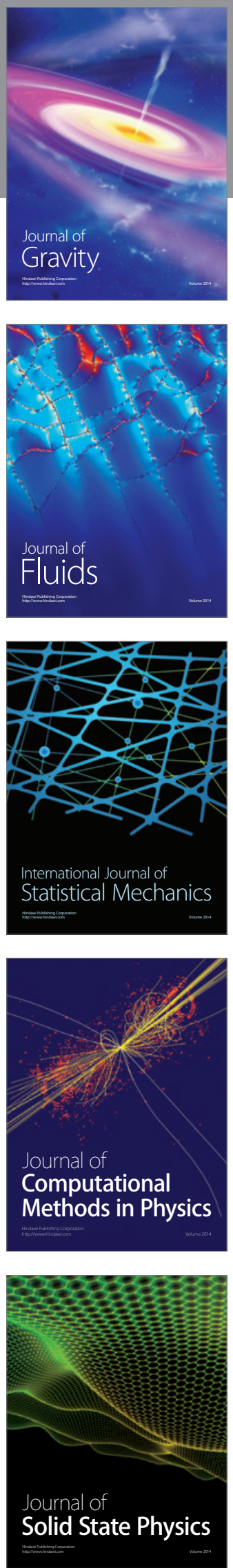

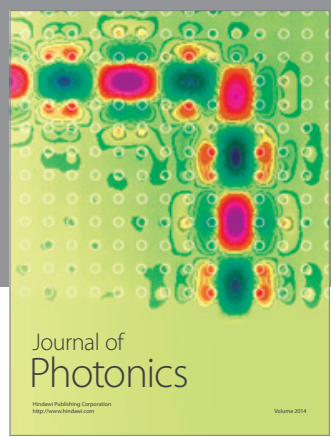

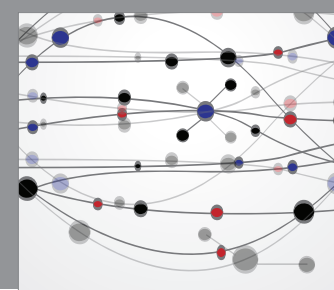

The Scientific World Journal

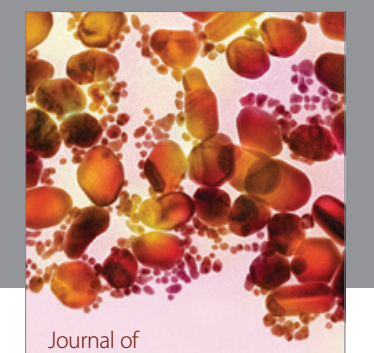

Soft Matter
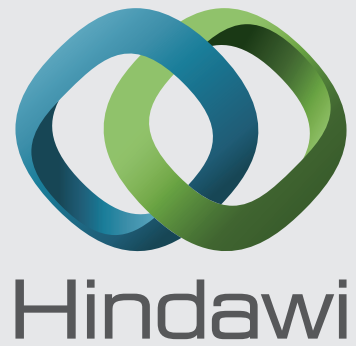

Submit your manuscripts at

http://www.hindawi.com
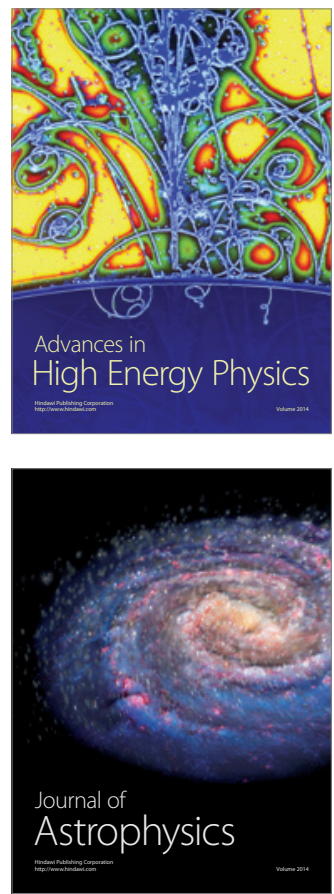
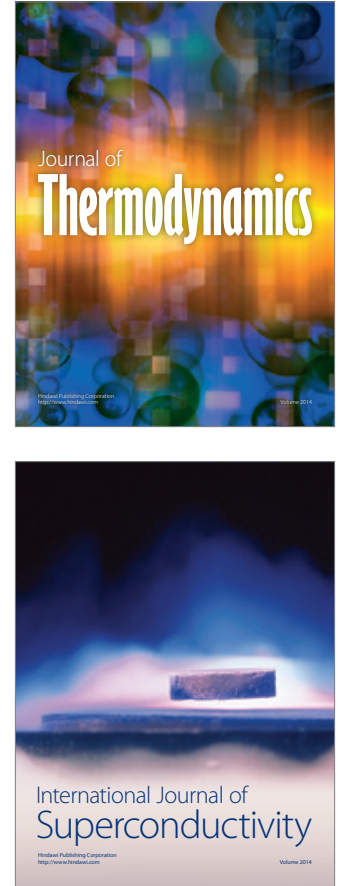
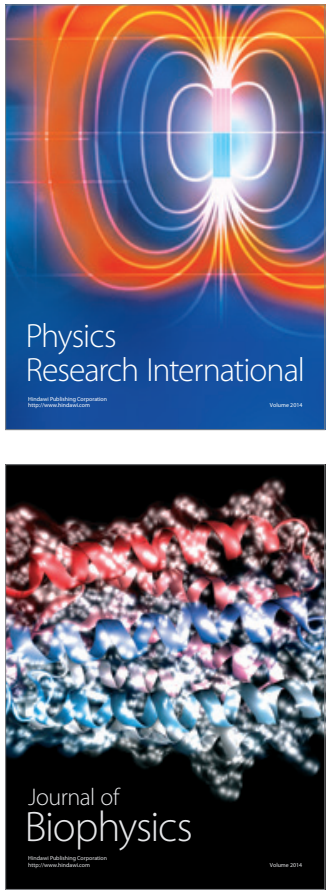
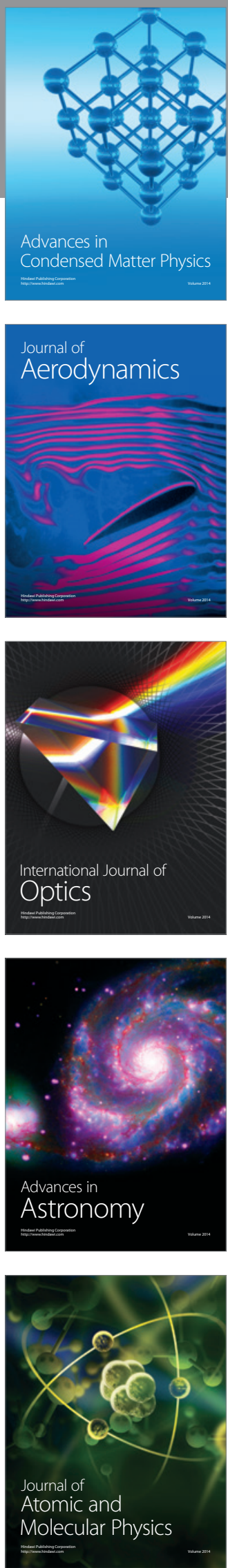